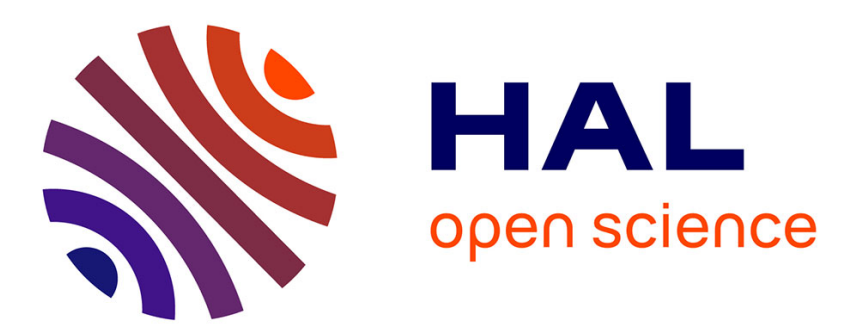

\title{
Finite element model of the impaction of a press-fitted acetabular cup
}

Adrien Michel, Vu-Hieu Nguyen, Romain Bosc, Romain Vayron, Philippe Hernigou, Salah Naili, Guillaume Haiat

\section{To cite this version:}

Adrien Michel, Vu-Hieu Nguyen, Romain Bosc, Romain Vayron, Philippe Hernigou, et al.. Finite element model of the impaction of a press-fitted acetabular cup . Medical and Biological Engineering and Computing, 2017, 55 (5), pp.781-791. 10.1007/s11517-016-1545-2 . hal-01785967

\section{HAL Id: hal-01785967 \\ https://hal.science/hal-01785967}

Submitted on 4 May 2018

HAL is a multi-disciplinary open access archive for the deposit and dissemination of scientific research documents, whether they are published or not. The documents may come from teaching and research institutions in France or abroad, or from public or private research centers.
L'archive ouverte pluridisciplinaire HAL, est destinée au dépôt et à la diffusion de documents scientifiques de niveau recherche, publiés ou non, émanant des établissements d'enseignement et de recherche français ou étrangers, des laboratoires publics ou privés. 


\section{Finite element model of the impaction of a press-fitted acetabular cup}

Adrien Michel ${ }^{1}$, Vu-Hieu Nguyen ${ }^{1}$, Romain Bosc $^{2}$, Romain Vayron$^{1}$, Philippe Hernigou ${ }^{3}$, Salah Naili ${ }^{1}$ and Guillaume Haiat ${ }^{1,4}$

${ }^{1}$ CNRS, Laboratoire de Modélisation et de Simulation Multi-Echelle, UMR CNRS 8208, France.

${ }^{2}$ Service de Chirurgie Plastique et Reconstructive, Hôpital Henri Mondor AP-HP, CHU Paris 12, Université Paris-Est, 51 avenue du Maréchal de Lattre de Tassigny, 94000 Créteil, France.

${ }^{3}$ Service de Chirurgie Orthopédique, Hôpital Henri Mondor AP-HP, CHU Paris 12, Université Paris-Est, 51 avenue du Maréchal de Lattre de Tassigny, 94000 Créteil, France.

${ }^{4}$ École de technologie supérieure, 1100 Notre-Dame Street West, Montreal, QC H3C 1K3, Canada; Research Center, Hôpital du Sacré-Cœur de Montréal, 5400, Gouin Boul. West, Montreal, QC H4J 1C5, Canada.

Submitted to Med Biol Eng Comput

Corresponding author:

Guillaume HAÏAT

Laboratoire de Modélisation et de Simulation Multi Echelle, UMR CNRS 8208,

61 avenue du Général de Gaulle,

94010 Créteil, France

tel : (33) 145171441

fax : (33) 145171433

e-mail : guillaume.haiat@univ-paris-est.fr

Total number of words: 4800

Number of words of the abstract: 198

Number of figures: 11

Number of tables: 1 
Finite element model of the impaction of a press-fitted acetabular cup: estimation of the contact area.

\begin{abstract}
Press-fit surgical procedures aim at providing primary stability to acetabular cup (AC) implants. Impact analysis constitutes a powerful approach to retrieve the AC implant insertion properties. The aim of this numerical study was to investigate the dynamic interaction occurring between the hammer, the ancillary and bone tissue during the impact and to assess the potential of impact analysis to retrieve AC implant insertion conditions.
\end{abstract}

A dynamic two-dimensional axisymmetric model was developed to simulate the impaction of the AC implant into bone tissue assuming friction at the bone-implant interface and large deformations. Different values of interference fit (from 0.5 to $2 \mathrm{~mm}$ ) and impact velocities (from 1 to $2 \mathrm{~m}_{\text {. }}{ }^{-1}$ ) were considered. For each configuration, the variation of the force applied between the hammer and the ancillary was analyzed and an indicator $I$ was determined based on the impact momentum of the signal.

The simulated results are compared to the experiments. The value of the polar gap decreases versus the impact velocity and increases versus the interference fit. The bone implant contact area was significantly correlated with the resonance frequency $\left(\mathrm{R}^{2}=0.94\right)$ and the indicator $\left(\mathrm{R}^{2}=0.95\right)$.

The results show the potential of impact analyses to retrieve the bone-implant contact properties.

Keywords: Acetabular cup; hip replacement; impact; finite element; bone; Biomechanics; bone

Author Biographies:

Adrien Michel has completed his PhD in biomechanics in the MSME laboratory. Romain Bosc and Philippe Hernigou are experienced surgeons. Vu-Hieu Nguyen and Salah Naili are experts in mechanical modeling and simulation. Romain Vayron and Guillaume Haiat are specialized in biomechanical engineering of implants. 
Finite element model of the impaction of a press-fitted acetabular cup: estimation of the contact area.

\section{Introduction}

Hip arthroplasty has become a standard surgical intervention [1]. However, failures still occur [2] and may have dramatic consequences such as pain, additional surgery and immobilization periods [3-5]. Aseptic loosening has been identified as one of the most current causes of implant failure [6, 7] and may be related to the implant primary stability, which is determinant for the quality of osseointegration phenomena [8]. A compromise must be found between i) a sufficient primary stability necessary to reduce the relative micromotions at the bone-implant interface, which may lead to the formation of fibrous tissue around the implant [9] and ii) excessive stresses around the implant, which may lead to bone tissue necrosis [10]. However, it remains difficult to assess the implant primary stability, which depends on the bone-implant contact area. In particular, surgeons use empirical approaches to assess the acetabular cup (AC) implant stability based on the noise produced by the impacts, which is not sufficient to estimate accurately the bone-implant contact area.

The mechanical interlocking of press-fitted AC implants within periacetabular bone plays an important role in the implant stability. The AC deformation after insertion should be minimized in order to allow proper insertion of the liner in the $\mathrm{AC}[11,12]$. The cup deformation was examined by changing implant designs or interference levels using static implicit loads [13] or explicit dynamics model [14] to simulate the implant impaction. The cup deformation was shown to increase with the level of interference fit $[13,14]$. In order to promote biological fixation through bone ingrowth and to minimize the gap between bone and the implant surface, different studies have also focused on finding an optimal configuration for the implant seating while considering different implant geometry, interference fit and friction levels $[15,16]$.

The AC implant stability has also been assessed in simulation studies through the measurement of relative micromotion occurring at the bone-implant interface during simulated gait cycles or others daily activities $[6,16,17]$ or using other biomechanical tests such as push-in [18], pull-out and edge loading tests [19]. Experimental methods have been developed to assess the femoral stem stability [20, 
Finite element model of the impaction of a press-fitted acetabular cup: estimation of the contact area.

21], loosening and insertion endpoint [22] using vibrational techniques. However, the aforementioned methods remain difficult to be employed intraoperatively and are restrained to the stem. More recently, our group has studied the time variation of the force applied between the hammer and the ancillary during impacts produced to insert the AC implant. The AC implant insertion obtained with reproducible mass drops could first be assessed by following the impact contact duration [23]. Then, the impact momentum was found to be a more precise indicator [24] of the implant status because it could predict the implant in vitro stability [25]. The approach was also validated by comparing the results obtained using an instrumented impact hammer and pull-out tests [26]. However, despite the development of a simple analytical model considering the AC implant as a flat punch [24], the physical phenomena responsible for the variation of the signal retrieved during such impacts remains unclear. A better understanding of the mechanical interaction occurring at the bone-implant interface would be of interest to improve the performance of the device under development.

The aim of this study is to improve the understanding of the biomechanical phenomena occurring during the $\mathrm{AC}$ implant impaction process and to validate the use of impact signal analysis to predict the bone-implant contact area. To do so, the impaction of the AC implant into a bone cavity was modeled using a two-dimensional axisymmetric finite element model. Different values of interference fit and impact velocity were used to obtain different insertion conditions and the simulated and experimental results were compared.

\section{Material and methods}

\subsection{Experimental configuration}

The present study used parameters derived from a previous experimental study [26] to simulate the insertion process of an AC implant into bone tissue. The reader is referred to [26] for further details. Briefly, a hemispherical cavity was machined at the upper surface of a bovine bone sample which was held in a clamp, as shown in Fig. 1(a). The AC implant was positioned so that its longitudinal axis was aligned with the cavity axis. The AC was then inserted into the cavity by performing a series of impacts 
Finite element model of the impaction of a press-fitted acetabular cup: estimation of the contact area.

with a hammer $(1.3 \mathrm{~kg})$. Then, an impact realized with a "moderate" energy (maximum force comprised between 2500 and $4500 \mathrm{~N}$ ) was made and the history of the force as a function of time $A(t)$ was recorded for each impact.

\subsection{Geometry and material properties}

A 2D-axisymmetric finite element model was employed to simulate the experiments described above. Figure 1(b) shows the geometrical configuration, which consists of three distinct media: the bone sample $\Omega_{b}$, the AC implant (merged with the ancillary) $\Omega_{c}$ and the hammer $\Omega_{h}$. The hammer $\Omega_{h}$ was designed by assembling two cylinders (radius $25.6 \mathrm{~mm}$ and height $75 \mathrm{~mm}$ ) and the sensor (radius $6 \mathrm{~mm}$ and height $20 \mathrm{~mm}$ ). The ancillary (cylinder with radius $8.5 \mathrm{~mm}$ and height $380 \mathrm{~mm}$ ) was glued to the AC to form $\Omega_{c}$. The AC implant had an outer radius of $25.5 \mathrm{~mm}$ and a thickness varying linearly as a function of the angle between 2.9 (at the cup dome) and $3.7 \mathrm{~mm}$ (at the cup rim), which corresponds to the properties of the implant used experimentally (Cerafit Uncemented Hip Prosthesis, Ceraver, Roissy, France) [26]. The bone sample was designed as a cylinder (radius $50 \mathrm{~mm}$ and height $40 \mathrm{~mm}$ ) with a hemispheric hole at the top of the sample with a radius varying between 49 and 50,5 mm, according to the interference fit level (see subsection 2.4).

In this study, all media were modeled as linear isotropic elastic materials, an assumption that will be discussed in section 4 . Table 1 shows the material properties used for each medium of the system. Mechanical properties of bone tissue were chosen similarly as what was done in [27]. The properties of the other materials were chosen accordingly to the materials used in [26].

\subsection{Numerical simulation method}

The numerical simulation is described in this section. The problem was solved using the Ansys software (ANSYS Inc., Canonsburg, PA, USA). 
Finite element model of the impaction of a press-fitted acetabular cup: estimation of the contact area.

\subsubsection{Configuration description.}

In order to simulate the dynamic behavior of the system (consisting of the three subdomains: $\Omega_{h}, \Omega_{c}$ and $\Omega_{b}$ ) due to impacts, nonlinear geometrical hypotheses need to be used. To express the problem in large displacement, it was necessary to distinguish the reference configuration at $t=0$ (in which the point coordinates are denoted by $\boldsymbol{X}$ ) and its current configuration at a given time $t$ (in which the point coordinates are denoted by $\boldsymbol{x}, \boldsymbol{x}=\boldsymbol{x}(\boldsymbol{X} ; t))$. The gradient and divergence operators with respect to $\boldsymbol{X}$ are denoted by $\operatorname{Grad}($.$) and \operatorname{Div}($.$) , respectively in what follows.$

\subsubsection{Deformation/displacement relations.}

The deformation gradient tensor is defined by:

$$
\boldsymbol{F}=\boldsymbol{I}+\operatorname{Grad}(\boldsymbol{u})
$$

where $\boldsymbol{u}(\boldsymbol{X} ; t)=\boldsymbol{x}-\boldsymbol{X}$ is the displacement vector and $\boldsymbol{I}$ is the 2nd-order identity tensor. By using the deformation gradient $\boldsymbol{F}$, the spatial velocity gradient tensor $\boldsymbol{L}$, the spatial rate of deformation tensor $\boldsymbol{D}$ and the spatial spin tensor $\boldsymbol{w}$ are defined by:

$$
\begin{gathered}
L=\dot{\boldsymbol{F}} \boldsymbol{F}^{-1}, \\
D=\frac{1}{2}\left(\boldsymbol{L}+\boldsymbol{L}^{\boldsymbol{T}}\right), \\
w=\frac{1}{2}\left(\boldsymbol{L}-\boldsymbol{L}^{\boldsymbol{T}}\right),
\end{gathered}
$$

\subsubsection{Linear momentum balance equation.}

By neglecting the applied body force, the movement of a point in any subdomain $\left(\Omega_{\alpha}\right.$, $\alpha=\{h, c, b\}$, may be described by the balance of linear momentum equation in terms of reference coordinates:

$$
\rho_{0} \ddot{\boldsymbol{u}}-\operatorname{Div} \boldsymbol{P}=\mathbf{0},
$$

where $\rho_{0}$ denotes the mass density at $t=0 ; \boldsymbol{u}(\boldsymbol{X} ; \mathrm{t})$ and $\ddot{\boldsymbol{u}}(\boldsymbol{X} ; \mathrm{t})$ are the displacement and acceleration vectors, respectively. $\boldsymbol{P}$ is the first Piola-Kirchhoff stress tensor, which is defined by: 
Finite element model of the impaction of a press-fitted acetabular cup: estimation of the contact area.

$$
\boldsymbol{P}=\operatorname{det}(\boldsymbol{F}) \boldsymbol{\sigma} \boldsymbol{F}^{-T}
$$

where $\sigma$ is the Cauchy stress tensor.

\subsubsection{Constitutive equation.}

The Hooke's law was used to describe the relation between stress increment and the strain increment. However, when considering the large rigid body rotation, the Cauchy stress is not objective and could be directly used in the constitutive equation. Here, a hypoelastic constitutive laws based on the Jaumann rate of the Cauchy stress are used and reads:

$$
\breve{\boldsymbol{\sigma}}=\lambda \operatorname{Tr}(\boldsymbol{D}) \boldsymbol{I}+2 \mu \boldsymbol{D}
$$

where $\lambda, \mu$ are two elastic constants; $\widetilde{\boldsymbol{\sigma}}$ is the Jaumann rate of the Cauchy stress :

$$
\breve{\sigma}=\dot{\sigma}-w \sigma+\sigma w
$$

which has been shown to be an objective quantity.

\subsubsection{Boundary and initial conditions.}

The boundary of each sub-domain is divided into different parts corresponding to the boundary nature: with imposed displacement $\Gamma_{\alpha}^{u}$, with axisymmetrical condition $\Gamma_{\alpha}^{\text {sym }}$; or with contact condition $\Gamma_{\alpha}^{c}$ as shown in Fig. 1. $\left(\Gamma_{\alpha}=\Gamma_{\alpha}^{u} U \Gamma_{\alpha}^{s y m} \mathrm{U} \Gamma_{\alpha}^{c}\right)$. For this system, only the bone $\Omega_{b}$ is fixed at its lower surface. No external forces are applied to the system. The movement of the system is governed by the impact from $\Omega_{h}$ on $\Omega_{c}$. The impact is initiated by a imposing a downward vertical velocity at $t=0$ to the hammer. At $t=0$, the bone and the implant are assumed to be at rest. The initial conditions read:

$$
\begin{array}{cc}
\boldsymbol{u}_{(t=0)}=\mathbf{0} & \text { in } \Omega_{\alpha} \\
\dot{\boldsymbol{u}}_{(t=0)}=\mathbf{0} & \text { in } \Omega_{c}, \Omega_{b} \\
\dot{\boldsymbol{u}}_{(t=0)}=\left(0,-\mathrm{V}_{0}\right)^{\mathrm{T}} & \text { in } \Omega_{h}
\end{array}
$$

The boundary conditions (except the contact conditions) read:

$$
\begin{array}{ll}
\boldsymbol{u}=\mathbf{0} & \text { in } \Gamma_{b}^{u} \\
\boldsymbol{u} \cdot e_{r}=0 & \text { in } \Gamma_{\alpha}^{s y m}
\end{array}
$$


Finite element model of the impaction of a press-fitted acetabular cup: estimation of the contact area.

\subsubsection{Contact conditions.}

The contact interaction at the interfaces are described using the Coulomb friction law, together with the Kuhn-Tucker condition. By denoting by $t_{N}$ and $t_{T}$ the normal and tangent components of the Piola traction, respectively; and by $g$ the gap function, the Kuhn-Tucker condition reads:

$$
\begin{gathered}
t_{N} \geq 0, \mathrm{~g} \leq 0, t_{N} g=0 \\
\left|t_{T}\right|-f t_{N} \leq 0
\end{gathered}
$$

where $f$ is the friction coefficient at the bone-implant interface. The value of $f$ was chosen equal to 0.3 , based on previous values used in the literature [16, 18, 28].

\subsection{Simulation protocol}

Different numerical simulations were carried out using the mechanical model described above. Various conditions were considered based on the experiments described in [26]. The simulation protocol can be divided into two successive stages.

The first phase (denoted "insertion stage" in what follows) includes $N$ successive impacts of $\Omega_{h}$ on $\Omega_{c}$, where the initial velocity of $\Omega_{h}$ is equal to $V_{0}$. The number $N$ of impacts comprised in the insertion phase was determined so that one additional impact leads to an AC implant additional insertion inferior to $1 \mu \mathrm{m}$. The time duration between the different impacts was chosen so that the system is at rest at the beginning of each new impact. At the end of each impact, the hammer velocity was reset at the prescribed value $V_{0}$.

The second phase (denoted "measurement stage" in what follows) starts after the insertion stage. It was composed by a unique impact of $\Omega_{h}$ on $\Omega_{c}$ occurring with an impact velocity equal to $0.4 \mathrm{~m} . \mathrm{s}^{-1}$, which corresponds to a relatively weak impact energy. This situation corresponds to what has been done experimentally [26].

Different configurations (including both insertion and measurement stages) were simulated with different values of impact velocity and of interference fit. The impact velocity was varied between [1 $\mathrm{m} . \mathrm{s}^{-1} ; 2 \mathrm{~m} . \mathrm{s}^{-1}$ ] by step of $0.25 \mathrm{~m} . \mathrm{s}^{-1}$ while the interference fit was varied in the interval [0.5 mm; $2 \mathrm{~mm}$ ] 
Finite element model of the impaction of a press-fitted acetabular cup: estimation of the contact area.

by step of $0.25 \mathrm{~mm}$, which was obtained by varying the cavity diameter between 49 and $50.5 \mathrm{~mm}$. The total number of simulation (including the insertion and measurement stages) was equal to 35 .

\subsection{Signal processing}

The variation of the force $A(t)$ applied between the hammer and the ancillary was determined for the impact realized during the measurement stage. A quantitative indicator $I$, referred to as impact momentum, was determined using:

$$
I=\frac{1}{A_{0} \cdot\left(t_{2}-t_{1}\right)} \int_{t_{1}}^{t_{2}} A(t) \cdot d t
$$

where $t_{1}=0.16 \mathrm{~ms}, t_{2}=0.31 \mathrm{~ms}$. The value of $A_{0}$ was arbitrarily set equal to $3500 \mathrm{~N}$ for normalization purposes in order to obtain values of the indicator $I$ comprised in the interval [0;1]. Changing the value of $A_{0}$ did not affect the results. The choice of the values $t_{1}$ and $t_{2}$ will be discussed in section 4 .

A frequency analysis of $A(t)$ was realized and the frequency for which the power spectrum reaches its maximum value was determined and referred to as resonance frequency in what follows. At the end of each procedure, the polar gap, defined by the distance between implant surface and bone tissue at the bottom of the AC implant was determined. Matlab (The Mathworks, Natick, MA, USA) was used to analyze all data.

\section{Results}

The mesh corresponding to the geometrical configuration described in Fig. 1 is shown in Fig. 2. This mesh resulted in approximately 1900 eight-nodded elements with quadratic behavior well suited to mesh an irregular geometry. Automatic time stepping was used for the computation with a time step comprised in the interval $[0.25 \mu \mathrm{s} ; 10 \mu \mathrm{s}]$. The impaction procedure allowed an impaction every $5 \mathrm{~ms}$ approximately. 
Finite element model of the impaction of a press-fitted acetabular cup: estimation of the contact area.

Figure 3 shows the vertical displacements of the hammer and of the ancillary (fixed to the AC implant) obtained for an interference fit of $1 \mathrm{~mm}$ and an impact velocity $V_{0}$ of $1.5 \mathrm{~m} \cdot \mathrm{s}^{-1}$. As shown in Fig. 3, the hammer bounces on the ancillary after each impact. Moreover, the vertical position of the ancillary (and of the AC implant) decreases after each impact. The difference of vertical position of the AC implant obtained before and after the impact decreases as a function of the impact number, which indicates that the AC implant insertion is more important at the beginning of the procedure. After around 18 impacts, the position of the AC implant weakly varies before and after the impact, which indicates that the AC implant is fully inserted into bone tissue.

Figure 4 shows the spatial variation of the Von-Mises stresses in the AC implant and in bone tissue after two, five and ten impacts realized during the insertion stage with an impact velocity of 1.5 $\mathrm{m} . \mathrm{s}^{-1}$ and an interference fit of $1 \mathrm{~mm}$. As shown in Fig. 4, the stresses are concentrated on the edge of the implant around the contact region, in particular after few impacts.

Figure 5 shows the evolution of the polar gap as a function of time during the entire simulation for an interference fit equal to $1 \mathrm{~mm}$ and different values of $V_{0}$. The polar gap decreases as function of time for all impact velocities and reaches a constant value when the insertion stage is finished.

Figure 6 summarizes the final value of the polar gap obtained at the end of the insertion stage for all values of the interference fit and of the impact velocity. As shown in Fig. 6, the value of the polar gap increases as a function of the interference fit for all values of the impact velocity. Moreover, the value of the polar gap decreases as a function of the impact velocity for all values of the interference fit except for $0.5 \mathrm{~mm}$, which can be explained by the fact that the AC implant is almost completely inserted in the bone cavity for all impact velocities.

Figure 7 a) (resp. b)) shows six simulated (resp. experimental) impact force signals obtained during the insertion phase of the AC implant with an interference fit of $1 \mathrm{~mm}$. The simulated signals 
Finite element model of the impaction of a press-fitted acetabular cup: estimation of the contact area.

corresponds to an impact velocity of $1.5 \mathrm{~m} \cdot \mathrm{s}^{-1}$. Figures $7 \mathrm{a}$ ) and b) show that the duration between the primary and secondary impact decreases as a function of the impact number.

Figure 8 a) shows the force applied between the hammer and the ancillary during the impact realized during the measurement phase with an interference fit of $1 \mathrm{~mm}$ and an impact velocity of $1 \mathrm{~m} \cdot \mathrm{s}^{-}$ ${ }^{1}$. Figure $8 \mathrm{~b}$ ) shows the displacements of the hammer and of the ancillary during the same impact. The initial contact occurs at $\mathrm{t}=0$ and lasts during $0.1 \mathrm{~ms}$ approximately. Secondary impacts are obtained between 0.24 and $0.39 \mathrm{~ms}, 0.43$ and $0.6 \mathrm{~ms}$ and 0.64 and $0.78 \mathrm{~ms}$. Figure 8 (c) and (d) show the same features for a procedure realized with the same interference fit of $1 \mathrm{~mm}$ but with an impact velocity of $1.5 \mathrm{~m} . \mathrm{s}^{-1}$. While the initial contact is similar, secondary impact occurs earlier for $V_{0}=1.5 \mathrm{~m} . \mathrm{s}^{-1}$ than for $V_{0}=1 \mathrm{~m} \cdot \mathrm{s}^{-1}$.

Figure 9 shows impact signals corresponding to the time variation of the force applied between the hammer and the ancillary for the impact realized during the measurement phase with an interference fit of $1 \mathrm{~mm}$ and different impact velocities. Figure 9 shows that the force signals of the measurement stage significantly vary as a function of the impact velocity.

Figure 10 shows the variation of the impact momentum $I$ derived from the signals shown in Fig. 9 as a function of the contact area when the impact velocity and the interference fit vary. A linear regression analysis shows a significant correlation $\left(\mathrm{R}^{2}=0.95\right)$ between $I$ and the contact area.

Figure 11 shows the variation of the resonance frequency as a function of the contact area when the values of the impact velocity and of the interference fit vary. A linear regression analysis shows a significant correlation $\left(\mathrm{R}^{2}=0.94\right)$ between contact area and the resonance frequency. 
Finite element model of the impaction of a press-fitted acetabular cup: estimation of the contact area.

\section{Discussion}

The originality of the present study is to provide more physical insight on the understanding of the phenomena occurring during impacts realized during the AC implant insertion, which is obtained using 2D axisymmetric finite element modeling.

The modeling approach described herein can first be validated by comparing our results with results obtained in the literature. Spears et al. [16] and Yew et al. [13] found a value of the polar gap equal to 0.4 and $0.17 \mathrm{~mm}$ respectively at the end of the insertion process for an interference fit of $1 \mathrm{~mm}$ using static modeling. Hothi et al. [14] found a value of the polar gap equal to $0.36 \mathrm{~mm}$ for an impact velocity equal to $1 \mathrm{~m} / \mathrm{s}$. These results are of the same order of magnitude compared to the ones found in the present paper, where a polar gap comprised between 0.35 and $1 \mathrm{~mm}$ is obtained after the AC insertion, according to the impact velocity (see Fig. 6). The difference with the literature can be explained by the different features (such as the shape of the ancillary, the AC diameter, bone geometry, the mode of impaction...) used in the different studies, which makes a precise quantitative comparison difficult.

As shown in Fig. 4, the highest values of stresses mainly occurred around the periphery of the $\mathrm{AC}$ implant at the beginning of the insertion. When the AC implant is progressively inserted into bone tissue, the stress field is distributed over a larger surface but the highest amplitude still remains at the AC periphery. This phenomenon is in agreement with previous results obtained in $[14,28]$ and can be explained by the interference fit which is responsible for the implant stability [29, 30].

Previous studies using either static $[13,16,27]$ or dynamic [14] analyses also found that the AC implant undergoes a "rebound" during the impaction (i.e. the AC implant is pushed into bone cavity and then bounces back due to the bone elastic recoil), a phenomenon due to residual stresses in bone tissue. However, this "rebound" is limited by frictional forces at the bone implant interface $[13,27]$. Similar results were found in the present study, as shown in Fig. 8. However, our study goes beyond previous 
Finite element model of the impaction of a press-fitted acetabular cup: estimation of the contact area.

findings since multiple rebounds of the hammer on the ancillary are evidenced (see Fig. 8), which constitutes a novelty.

The duration between the first and secondary impacts is shown to decrease i) during the insertion phase when the impact number increases (see Fig. 7) and ii) during the measurement phase when the impact velocity increases (see Fig. 8 and 9). The decrease of the time between the first and secondary impact can be explained by the decrease of the polar gap obtained i) during the insertion phase and ii) when the impact velocity increases (see Fig. 6), which induces a lower time allocated to the AC implant to impact the bottom of the cavity.

Although the behavior of the experimental and simulated impact signals shown in Fig. 7 is qualitatively similar, some differences can be observed when comparing Figs. 7 a) and b). In particular, the experimental secondary impacts present lower amplitudes and frequency content compared to the simulated results. The difference between the simulated and experimental results may be explained by the following limitations of the numerical approach, including assumptions made on the geometrical configuration, bone material properties and the modeling of the bone-implant interface described below. Note that these assumptions have already been made in all previous approaches carried out in the literature $[13,16,27]$.

First, the geometrical configuration differs between the experimental and numerical approaches. The experimental configuration consisted in inserting the AC implant in the proximal part of a bovine femur (which is composed of trabecular bone surrounded by cortical bone) fixed in a resin, as shown in Fig. 1a). This complex geometrical configuration was modeled as a cylinder of trabecular bone with a diameter of $100 \mathrm{~mm}$ and a height of $50 \mathrm{~mm}$ with fixed boundary conditions at the bottom of the cylinder (see Fig. 1b)). The experimental configuration is less stiff compared to the numerical configuration due to the absence of the resin and to the lesser amount of trabecular bone in the simulations. We choose to consider a simplified geometrical configuration because our aim was to understand physical phenomena occurring during the impacts. Despite these limitations, the same behavior of the time difference 
Finite element model of the impaction of a press-fitted acetabular cup: estimation of the contact area.

obtained between the two first impacts were obtained experimentally and numerically, which indicates that the model was able to capture the mechanisms occurring during the impacts. Moreover, we verified that increasing the height of the cylinder induces a decrease of the frequency content of the secondary impact but does not affect the behavior of the impact signals (data not shown).

Second, bone properties were chosen isotropic, homogeneous and elastic, similarly as what has been done in previous studies focusing on the biomechanical behavior of the AC implant $[13,14]$. Such approximation corresponds to a simple modeling of the acetabular bone biomechanical properties. In particular, bone properties are known to be anisotropic and heterogeneous around the AC implant. The main trabecular orientation is expected to be perpendicular to the AC implant surface due to the presence of cartilage which induce very low friction rate in normal conditions. Moreover, bone is known to exhibit viscoelastic properties, which are likely to influence the dynamic behavior of the studied system. In particular, the higher amplitude of the secondary impact obtained in the simulated results may be explained by the elastic modeling of bone properties which does not account for dissipation mechanisms. However, the precise bone properties should be determined in a patient specific manner, which is out of scope of the present approach since we choose not to investigate the influence of bone properties on the impact signals. We verified that decreasing the value of the Young's modulus of trabecular bone induces a decrease of the frequency content of the secondary impact but does not affect the behavior of the impact signals (data not shown).

Third, the modeling of the bone-implant interface is relatively simple since a classical friction behavior is considered using the Coulomb's law, which corresponds to a simple configuration used by all other studies investigating the behavior of the $\mathrm{AC}$ implant in bone tissue [13, 16, 27]. In particular, the actual bone-implant contact ratio is typically around $60 \%$, while we considered a fully bone-implant interface. Moreover, plastic deformations are also likely to occur in bone tissue around the interface, which may affect its mechanical behavior. The partial bone-implant contact, together with plastic deformations, lead to the possibility of extracting a macroscopic constitutive law for the bone-implant interface based on its microstructural features, which may also explain the higher rigidity of the system 
Finite element model of the impaction of a press-fitted acetabular cup: estimation of the contact area.

predicted by the numerical simulation compared to the experimental results. However, the modeling of the bone-implant interface remains an open issue that should be solved to better understand the behavior of this system.

In the present paper, the impact momentum $I$ was devised following the results obtained in previous studies [24-26]. However, several parameters were modified to take into account the specific configuration described herein. In particular, the value of $t_{l}$ was chosen in order to include the beginning of each secondary impact and the choice of $t_{2}$ comes from the differences between the force signals. An optimization study was carried out in order to maximize the correlation coefficient between $I$ and the bone implant contact area. The results were not significantly affected by changing the value of $t_{l}$ between $0.16 \mathrm{~ms}$ and $0.20 \mathrm{~ms}$ and of $t_{2}$ between 0.29 and $0.33 \mathrm{~ms}$ (less than $3 \%$ difference for $\mathrm{R}^{2}$, data not shown).

The results obtained in Fig. 10 indicate that the impact momentum is correlated to the contact area, which is an important parameter for the implant success $[6,30]$. These results indicate that the impact momentum may constitute an important parameter to determine the insertion condition of the AC implant because it is related to the contact area. The results shown in Fig. 11 shows that the model predicts an increase of the resonance frequency as a function of the contact area. This results is in agreement with the empirical analytical model developed in [24] which assumes a flat punch configuration.

As expected, increasing the impact velocity for the same interference fit induces a decrease of the number of impacts necessary to insert the AC implant. However, similar insertion conditions could be obtained with different impact velocity. For instance, for an interference fit of $1 \mathrm{~mm}$, similar final polar gap of around $380 \mu \mathrm{m}$ could be obtained after 8 impacts at $2 \mathrm{~m} \cdot \mathrm{s}^{-1}, 12$ impacts $1,75 \mathrm{~m} \cdot \mathrm{s}^{-1}$ or 18 impacts at $1,5 \mathrm{~m} \cdot \mathrm{s}^{-1}$. A compromise must be found by the surgeon between a sufficiently high value of $V_{0}$ in order to reduce the number of impacts and a sufficiently low value of $V_{0}$ in order to decrease the 
Finite element model of the impaction of a press-fitted acetabular cup: estimation of the contact area.

fracture risk. Such compromise also depends on bone quality, which is considered constant in the present study (see below).

Previous works [13, 31-33] have shown that the value of the polar gap at the end of the insertion should be lower than around $350 \mu \mathrm{m}$ to allow appropriate osseointegration. In order to reach this requirement, our results indicate that the interference fit should be strictly below $1 \mathrm{~mm}$ or equal to $1 \mathrm{~mm}$ with an impact velocity equal to $2 \mathrm{~m} \cdot \mathrm{s}^{-1}$. Hence, another compromise has to be found by the surgeon between an interference fit level sufficiently high to ensure an adequate primary stability and sufficiently low to induce a polar gap value low enough to allow appropriate osseointegration.

\section{Conclusion}

The results obtained herein show the potential of the impact momentum to provide useful information on the implant insertion. When considering a force sensor included into the surgical hammer, the technique presents the advantage of being easily integrated into the operating room. Compared to vibrational techniques [20,34], the present approach is easier to handle and does not require additional time for the surgery. However, important works are necessary before going into the clinic. In particular, the modeling approach should be improved by considering a better description of the geometrical configuration, of bone properties as well as of the bone-implant interface. However, despite these limitations, the developed numerical model was able to capture the physical determinants of the phenomena occurring during the implant impaction. Moreover, the method should be tested experimentally in human cadavers, a study being now under way [35].

\section{Acknowledgements}

This work has been supported by French National Research Agency (ANR) through the PRTS program (project OsseoWave ANR-13-PRTS-0015). 
Finite element model of the impaction of a press-fitted acetabular cup: estimation of the contact area.

\section{References}

[1] H. Sun, H. Inaoka, Y. Fukuoka, T. Masuda, A. Ishida, and S. Morita, "Range of motion measurement of an artificial hip joint using CT images," Med Biol Eng Comput, vol. 45, pp. 1229-35, Dec 2007.

[2] J. S. Wu, S. L. Hsu, and J. H. Chen, "Evaluating the accuracy of wear formulae for acetabular cup liners," Med Biol Eng Comput, vol. 48, pp. 157-65, Feb 2010.

[3] N. Piolanti, L. Andreani, P. D. Parchi, E. Bonicoli, F. Niccolai, and M. Lisanti, "Clinical and radiological results over the medium term of isolated acetabular revision," ScientificWorldJournal, vol. 2014, p. 148592, 2014.

[4] R. P. Shah, J. A. Scolaro, R. Componovo, J. P. Garino, and G. C. Lee, "Ceramic-on-ceramic total hip arthroplasty in patients younger than 55 years," J Orthop Surg (Hong Kong), vol. 22, pp. 338-41, Dec 2014.

[5] Y. M. Baghdadi, A. N. Larson, and R. J. Sierra, "Long-term results of the uncemented acetabular component in a primary total hip arthroplasty performed for protrusio acetabuli: a fifteen year median follow-up," Int Orthop, Nov 92014.

[6] J. T. Hsu, C. H. Chang, H. L. Huang, M. E. Zobitz, W. P. Chen, K. A. Lai, et al., "The number of screws, bone quality, and friction coefficient affect acetabular cup stability," Med Eng Phys, vol. 29, pp. 1089-95, Dec 2007.

[7] K. A. Fehring, J. R. Owen, A. A. Kurdin, J. S. Wayne, and W. A. Jiranek, "Initial stability of pressfit acetabular components under rotational forces," J Arthroplasty, vol. 29, pp. 1038-42, May 2014.

[8] H. C. Lin, W. M. Chi, Y. J. Ho, and J. H. Chen, "Effects of design parameters of total hip components on the impingement angle and determination of the preferred liner skirt shape with an adequate oscillation angle," Med Biol Eng Comput, vol. 51, pp. 397-404, Apr 2013.

[9] J. T. Hsu, K. A. Lai, Q. Chen, M. E. Zobitz, H. L. Huang, K. N. An, et al., "The relation between micromotion and screw fixation in acetabular cup," Comput Methods Programs Biomed, vol. 84, pp. 34-41, Oct 2006.

[10] M. J. Curtis, R. H. Jinnah, V. D. Wilson, and D. S. Hungerford, "The initial stability of uncemented acetabular components," Journal of Bone and Joint Surgery-British Volume, vol. 74, pp. 372376, May 1992.

[11] M. C. Bone, P. Dold, M. Flohr, R. Preuss, T. J. Joyce, D. Deehan, et al., "A novel method for measuring acetabular cup deformation in cadavers," Proc Inst Mech Eng H, vol. 227, pp. 13414, Dec 2013.

[12] M. Squire, W. L. Griffin, J. B. Mason, R. D. Peindl, and S. Odum, "Acetabular component deformation with press-fit fixation," J Arthroplasty, vol. 21, pp. 72-7, Sep 2006.

[13] A. Yew, Z. M. Jin, A. Donn, M. M. Morlock, and G. Isaac, "Deformation of press-fitted metallic resurfacing cups. Part 2: Finite element simulation," Proc Inst Mech Eng H, vol. 220, pp. 311-9, Feb 2006.

[14] H. S. Hothi, J. J. Busfield, and J. C. Shelton, "Explicit finite element modelling of the impaction of metal press-fit acetabular components," Proc Inst Mech Eng H, vol. 225, pp. 303-14, Mar 2011.

[15] K. L. Ong, J. Lehman, W. I. Notz, T. J. Santner, and D. L. Bartel, "Acetabular cup geometry and bone-implant interference have more influence on initial periprosthetic joint space than joint loading and surgical cup insertion," J Biomech Eng, vol. 128, pp. 169-75, Apr 2006.

[16] I. R. Spears, M. M. Morlock, M. Pfleiderer, E. Schneider, and E. Hille, "The influence of friction and interference on the seating of a hemispherical press-fit cup: a finite element investigation," J Biomech, vol. 32, pp. 1183-9, Nov 1999.

[17] I. Udofia, F. Liu, Z. Jin, P. Roberts, and P. Grigoris, "The initial stability and contact mechanics of a press-fit resurfacing arthroplasty of the hip," J Bone Joint Surg Br, vol. 89, pp. 549-56, Apr 2007. 
Finite element model of the impaction of a press-fitted acetabular cup: estimation of the contact area.

[18] R. Ghosh, S. Gupta, A. Dickinson, and M. Browne, "Experimental validation of finite element models of intact and implanted composite hemipelvises using digital image correlation," J Biomech Eng, vol. 134, p. 081003, Aug 2012.

[19] C. Zietz, A. Fritsche, D. Kluess, W. Mittelmeier, and R. Bader, "Influence of acetabular cup design on the primary implant stability : an experimental and numerical analysis," Orthopade, vol. 38, pp. 1097-105, Nov 2009.

[20] L. C. Pastrav, S. V. Jaecques, I. Jonkers, G. V. Perre, and M. Mulier, "In vivo evaluation of a vibration analysis technique for the per-operative monitoring of the fixation of hip prostheses," J Orthop Surg Res, vol. 4, p. 10, 2009.

[21] E. Varini, E. Bialoblocka-Juszczyk, M. Lannocca, A. Cappello, and L. Cristofolini, "Assessment of implant stability of cementless hip prostheses through the frequency response function of the stem-bone system," Sensors and Actuators a-Physical, vol. 163, pp. 526-532, Oct 2010.

[22] L. C. Pastrav, S. V. Jaecques, M. Mulier, and G. Van Der Perre, "Detection of the insertion end point of cementless hip prostheses using the comparison between successive frequency response functions," J Appl Biomater Biomech, vol. 6, pp. 23-9, Jan-Apr 2008.

[23] V. Mathieu, A. Michel, C. H. Flouzat Lachaniette, A. Poignard, P. Hernigou, J. Allain, et al., "Variation of the impact duration during the in vitro insertion of acetabular cup implants," Med Eng Phys, vol. 35, pp. 1558-63, Nov 2013.

[24] A. Michel, R. Bosc, V. Mathieu, P. Hernigou, and G. Haiat, "Monitoring the press-fit insertion of an acetabular cup by impact measurements: Influence of bone abrasion," Proc Inst Mech Eng H, vol. 228, pp. 1027-34, Oct 2014.

[25] A. Michel, R. Bosc, R. Vayron, and G. Haiat, "In vitro evaluation of the acetabular cup primary stability by impact analysis," J Biomech Eng, Jan 12015.

[26] A. Michel, R. Bosc, R. Vayron, and G. Haiat, "Ex vivo estimation of cementless acetabular cup stability using an impact hammer," Med Eng Phys, submitted.

[27] Z. M. Jin, S. Meakins, M. M. Morlock, P. Parsons, C. Hardaker, M. Flett, et al., "Deformation of press-fitted metallic resurfacing cups. Part 1: Experimental simulation," Proc Inst Mech Eng $H$, vol. 220, pp. 299-309, Feb 2006.

[28] D. Janssen, R. E. Zwartele, H. C. Doets, and N. Verdonschot, "Computational assessment of press-fit acetabular implant fixation: the effect of implant design, interference fit, bone quality, and frictional properties," Proc Inst Mech Eng H, vol. 224, pp. 67-75, 2010.

[29] E. Adler, S. A. Stuchin, and F. J. Kummer, "Stability of press-fit acetabular cups," J Arthroplasty, vol. 7, pp. 295-301, Sep 1992.

[30] J. R. MacKenzie, J. J. Callaghan, D. R. Pedersen, and T. D. Brown, "Areas of contact and extent of gaps with implantation of oversized acetabular components in total hip arthroplasty," Clin Orthop Relat Res, pp. 127-36, Jan 1994.

[31] L. Carlsson, T. Rostlund, B. Albrektsson, and T. Albrektsson, "Implant fixation improved by close fit. Cylindrical implant-bone interface studied in rabbits," Acta Orthop Scand, vol. 59, pp. 2725, Jun 1988.

[32] P. M. Sandborn, S. D. Cook, W. P. Spires, and M. A. Kester, "Tissue response to porous-coated implants lacking initial bone apposition," J Arthroplasty, vol. 3, pp. 337-46, 1988.

[33] M. Baleani, R. Fognani, and A. Toni, "Initial stability of a cementless acetabular cup design: experimental investigation on the effect of adding fins to the rim of the cup," Artif Organs, vol. 25, pp. 664-9, Aug 2001.

[34] E. Varini, M. Vandi, L. Cristofolini, A. Cappello, and A. Toni, "Intra-operative tests on cementless hip stem mechanical stability," Journal of Mechanics in Medicine and Biology, vol. 6, pp. 25-34, Mar 2006.

[35] A. Michel, R. Bosc, R. Vayron, and G. Haiat, " Assessing the acetabular cup implant primary stability by impact analysis: a cadaveric study.," PLOS One, submitted. 
Finite element model of the impaction of a press-fitted acetabular cup: estimation of the contact area.

\section{Figure legends}

Fig. 1: Representation of (a) the experimental set-up and (b) simulation domains.

Fig. 2 2D axisymmetric meshing of the geometrical configuration

Fig. 3 Position of the hammer impacting surface (solid line) and the ancillary top surface (dashed line) obtained during the impaction with an interference fit of $1 \mathrm{~mm}$ and an impact velocity of $1.5 \mathrm{~m} . \mathrm{s}^{-1}$. The origin $(z=0)$ of the ordinate axis corresponds to the position of the bottom of the bone hole.

Fig. 4 Von-Mises stresses in the AC implant and in periacetabular bone after (a) two, (b) five and (c) ten impacts for an impact velocity of $1.5 \mathrm{~m} \cdot \mathrm{s}^{-1}$ and an interference fit of $1 \mathrm{~mm}$.

Fig. 5 Variation of the polar gap during the insertion phase realized with different impact velocities and an interference fit equal to $1 \mathrm{~mm}$.

Fig. 6 Polar gap obtained at the end of each impaction process as a function of the interference fit for different values of impact velocities. The grey dashed line corresponds to a threshold of $350 \mu \mathrm{m}$ representing an adequate insertion of the AC implant [31-33].

Fig. 7 Simulated (a) and experimental (b) $r f$ signals corresponding to the force measured during different impacts of the AC implant insertion procedure with an interference fit of $1 \mathrm{~mm}$. The simulated signals corresponds to an impact velocity of $1.5 \mathrm{~m} \cdot \mathrm{s}^{-1}$.

Fig. 8 Description of the impact realized during the measurement phase. (a) (respectively (c)): Force between the hammer and the ancillary obtained for an interference fit of $1 \mathrm{~mm}$ and an impact velocity of $1 \mathrm{~m} . \mathrm{s}^{-1}$ (resp. $\left.1.5 \mathrm{~m} . \mathrm{s}^{-1}\right)$. (b) (respectively (d)): Vertical displacement of the hammer and of the ancillary for an interference fit of $1 \mathrm{~mm}$ and an impact velocity of $1 \mathrm{~m} \cdot \mathrm{s}^{-1}$ (resp. $1.5 \mathrm{~m} \cdot \mathrm{s}^{-1}$ ).

Fig. 9 Five signals corresponding to the time variation of the force applied between the hammer and the ancillary for the impact realized during the measurement phase for different values of the impact velocity and an interference fit equal to $1 \mathrm{~mm}$.

Fig. 10 Variation of the impact momentum $I$ as a function of the contact area for different values of the interference fit and of the impact velocity.

Fig. 11 Variation of the resonance frequency as a function of the contact area for different values of the interference fit and of the impact velocity. 
Finite element model of the impaction of a press-fitted acetabular cup: estimation of the contact area.
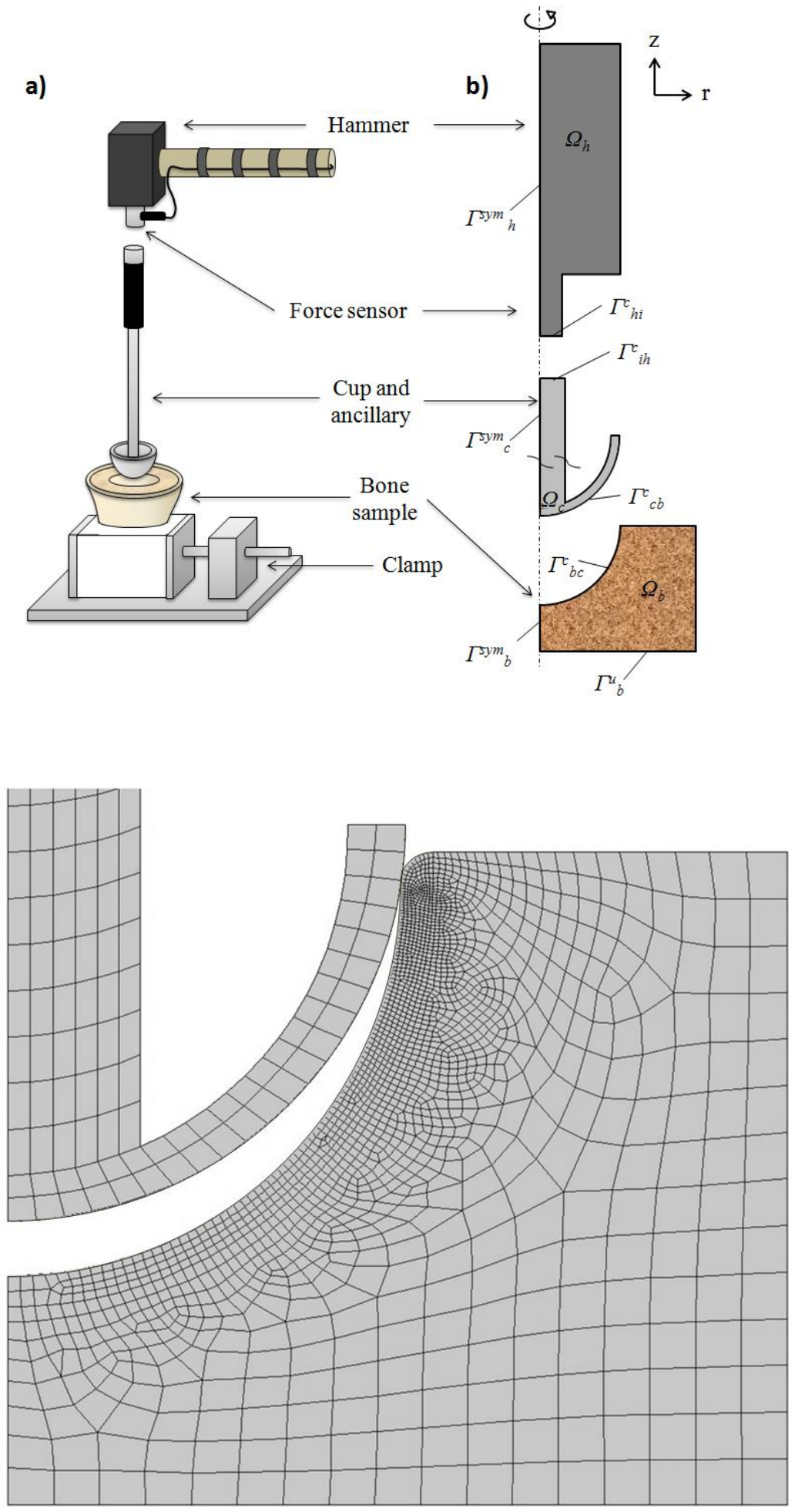
Finite element model of the impaction of a press-fitted acetabular cup: estimation of the contact area.
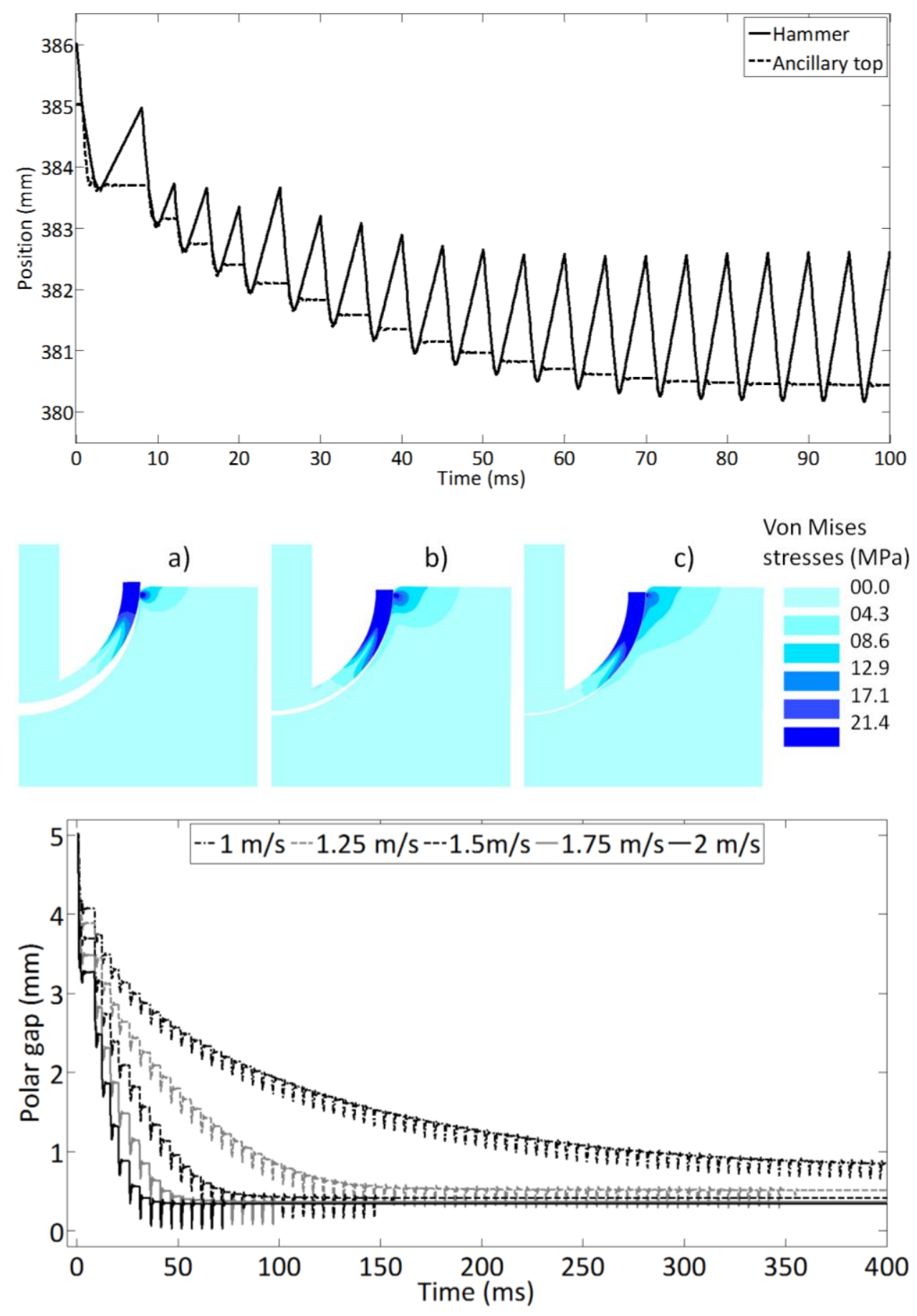
Finite element model of the impaction of a press-fitted acetabular cup: estimation of the contact area.
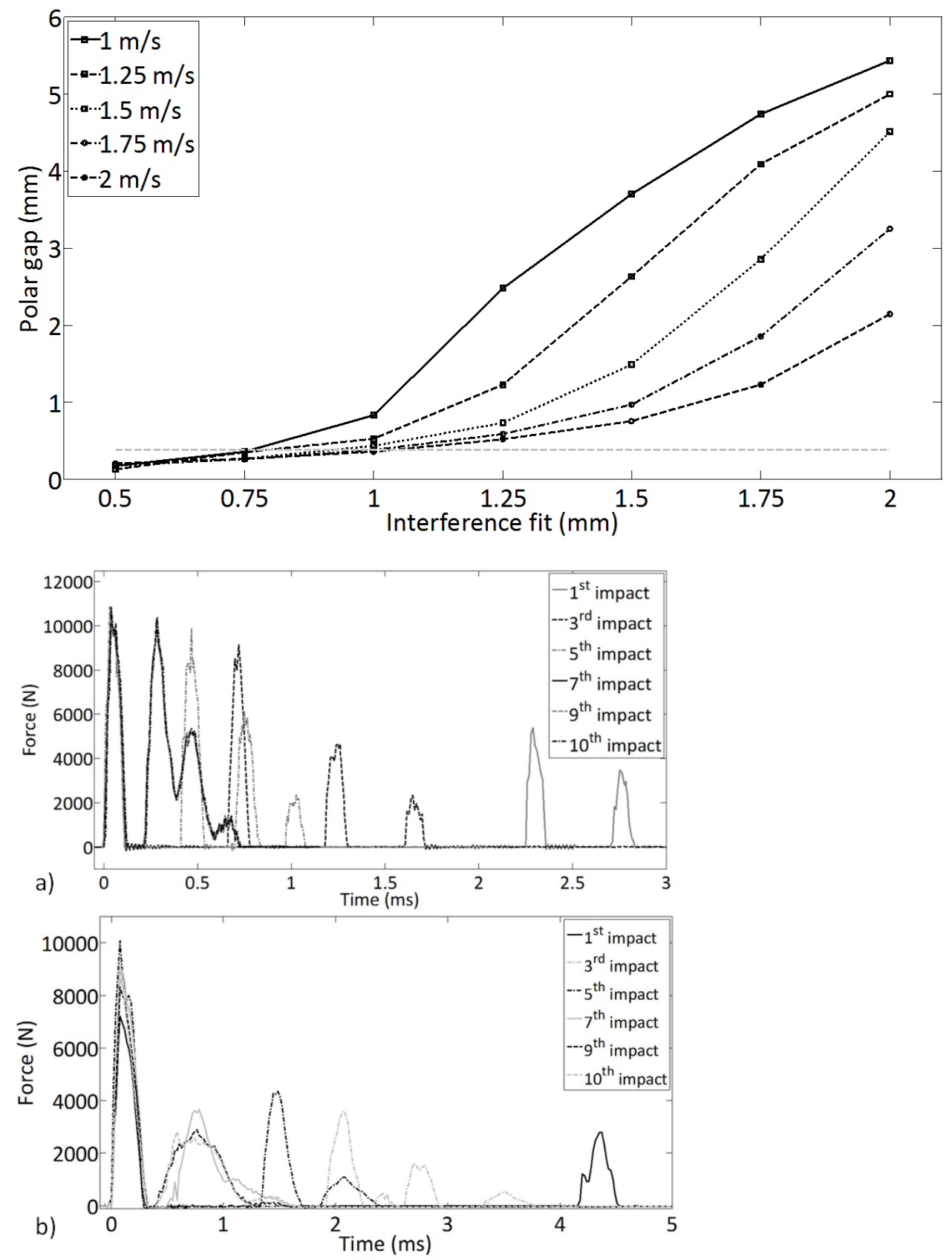
Finite element model of the impaction of a press-fitted acetabular cup: estimation of the contact area.
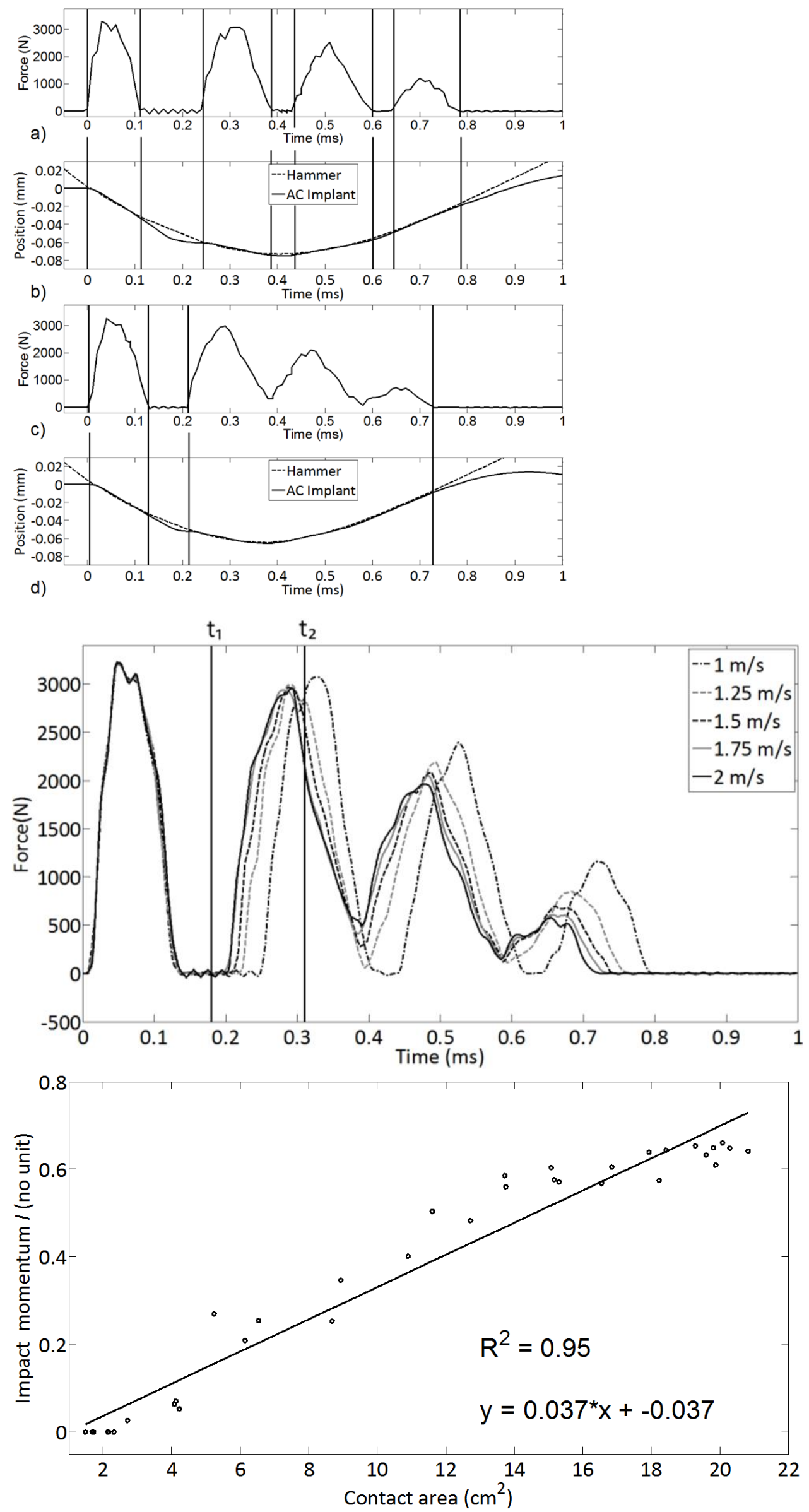
Finite element model of the impaction of a press-fitted acetabular cup: estimation of the contact area.

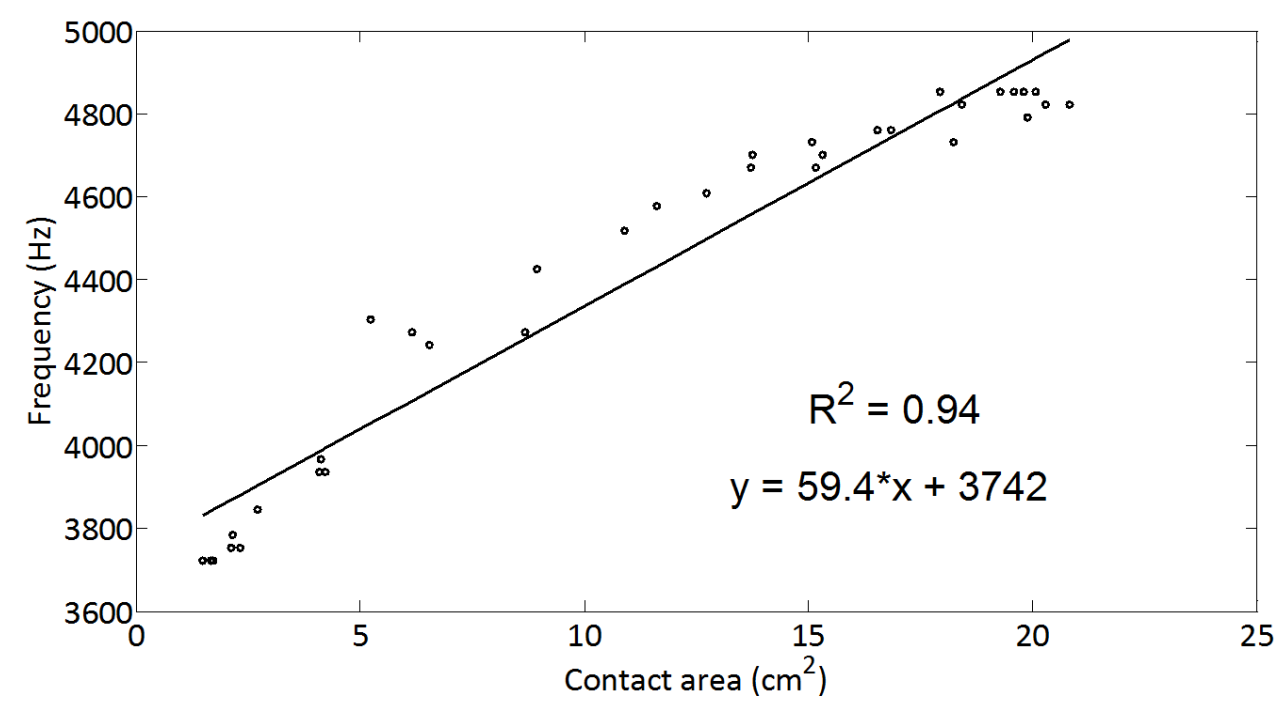

